\title{
La nueva gestión pública y las políticas de igualdad de género en las universidades
}

\author{
Cecilia CASTAÑO COLLADO \\ Universidad Complutense de Madrid y GENTIC (UOC) \\ ccastano@ucm.es
}

Recibido: Septiembre 2016

Aceptado: Diciembre 2016

\begin{abstract}
RESUMEN
Las políticas de igualdad de género en las universidades europeas se han aplicado en un contexto de reformas orientadas a la implantación de la lógica del mercado en la gestión de la educación superior (New Public Management). Mayor transparencia y mecanismos de competitividad empresarial podrían poner en cuestión los sistemas tradicionales de producción y reproducción del poder masculino en las universidades. Este artículo analiza la literatura sobre la experiencia de algunos países que nos han precedido en la implantación de políticas de igualdad en paralelo con políticas de transparencia y competitividad con el objetivo de extraer consecuencias que sean útiles para las políticas públicas.
\end{abstract}

Palabras clave: New Public Mangement, género y ciencia, igualdad de género en la Ciencia, Educación superior.

\section{The New Public Management and gender equality policies in Universities}

\begin{abstract}
Gender equality policies at European universities have been introduced in the context of reforms of higher education aimed at the implementation of market logic in the management of higher education (New Public Management). Greater transparency and mechanisms of competitiveness could call into question the traditional systems of production and reproduction of male power in universities. This article analyzes the experience of some countries that have preceded Spain in the implementation of equality policies in parallel with policies of transparency and competitiveness. Our goal is to draw conclusions that are useful for public policy.
\end{abstract}

Keywords: New Public Mangement, Gender and Science, Policies for Gender Equality in Science, Higher Education.

\section{INTRODUCCIÓN}

En las últimas décadas se han puesto en marcha numerosas políticas orientadas a fomentar la igualdad de género en la educación superior y en la investigación académica, tanto en los países del ámbito Anglo-Sajón, como en los países más 
avanzados del Centro, el Norte y el Sur de Europa (Addis et al., 2012; Müller et al., 2011; Rees, 2002). La Estrategia de Lisboa (Consejo Europeo, 2000) y el Programa Horizonte 2020 de la Comisión Europea han reforzado estas iniciativas al urgir a los Estados Miembros a impulsar la igualdad de género en la investigación como herramienta para atraer el mejor talento investigador y convertir la Unión Europea en la economía del conocimiento más dinámica y competitiva del mundo.

Aunque se han realizado avances importantes, que se reflejan en el aumento del número de profesoras universitarias e investigadoras, se advierte una situación de estancamiento que provoca frustración entre las mujeres porque su presencia es escasa en los niveles más altos y elevada en los más bajos de la educación superior, y el equilibrio de género no se alcanza en los ámbitos de toma de decisiones (European Commission, 2015). La decepción por los frutos de las políticas de igualdad de género pueden achacarse tanto a un diseño inadecuado de las mismas (la brecha entre la retórica de los principios enunciados, los objetivos perseguidos y la efectividad de los instrumentos diseñados), como a las dificultades que encuentra su aplicación en instituciones universitarias con culturas y prácticas sesgadas por género.

También en las últimas décadas, los países europeos han llevado a cabo reformas de las instituciones de educación superior bajo la influencia del New Public Management, que introduce estrategias de gestión empresarial -con mecanismos de transparencia, evaluación de la productividad y la calidad- en las relativamente independientes y autogobernadas áreas de conocimiento, departamentos y cátedras de las universidades e instituciones de investigación. Los nuevos mecanismos se superponen a los anteriores sistemas de excelencia académica y meritocracia, que rigen el funcionamiento de las instituciones universitarias y científicas. Y podrían favorecer la equidad de género si consideramos que la captación del talento femenino inspira, al menos parcialmente, la Estrategia 2020 de la UE.

Aunque la igualdad de género es más una cuestión de equidad que de eficiencia, cabría esperar que los principios de excelencia académica y meritocracia, reforzados por mecanismos de transparencia y unidos a políticas de inclusión de las mujeres (lo que se ha llamado inclusive excellence, Zippel et al., 2016), favoreciesen la igualdad efectiva de género. Sin embargo, en las universidades de hoy persisten la opacidad de los sistemas de selección y promoción, el sesgo de género en la evaluación de méritos y la infravaloración de la enseñanza frente a la investigación, que se añaden a las dificultades para conciliar trabajo y vida privada. Todos estos factores contribuyen a la persistencia de un clima de sexismo institucionalizado y ausencia de conciencia de la desigualdad de género. A pesar de las reformas y reestructuraciones con criterios de mercado, los cambios culturales claves para el avance de la igualdad de género son los más difíciles de establecer, porque ponen en cuestión el sistema patriarcal vigente en las universidades.

El objetivo de este artículo es contribuir al debate sobre la efectividad de las políticas de igualdad de género en las instituciones universitarias y científicas, reuniendo aportaciones interesantes de la literatura y la investigación de género que reflejan las experiencias de los países que han sido pioneros en este ámbito. Forma parte del Proyecto GENERA TALENTO (Plan Estatal I+D+i, 2013) del que la autora es 
investigadora principal, que analiza la aplicación de las políticas de igualdad en las universidades españolas. Lo que se expondrá a continuación es parte de la revisión de la literatura académica internacional llevada a cabo para dicho proyecto $\mathrm{y}$, concretamente, aquéllas aportaciones centradas en el impacto de las políticas de reforma universitaria inspiradas en el New Public Management sobre el avance de las mujeres y la efectividad de las políticas de igualdad de género. Para realizar dicha revisión se ha tomado como punto de partida el Meta-Análisis sobre Género en la Ciencia y la Investigación (Caprile, 2012), y más concretamente el informe temático sobre Políticas para la igualdad de género en la ciencia y la investigación (Castaño et al., 2010). A continuación se han rastreado los papers y contribuciones aportados a las conferencias bianuales Gender in Higher Education, sobre la temática objeto del proyecto, lo que ha permitido acceder a un considerable volumen de artículos, presentaciones, proyectos e informes de investigación que tienen como objetivo el análisis de las políticas de igualdad de género en las universidades en distintos países y consideran el New Public Management como uno de los elementos clave que afecta tanto al diseño de las políticas como al éxito o fracaso de su aplicación. La revisión aportada en este artículo no pretende ser exhaustiva y abarca fundamentalmente las experiencias de los países pioneros - del ámbito anglosajón- y de los que son hoy más proactivos -Alemania y Austria- en la temática objeto de estudio.

\section{LAS POLÍTICAS DE IGUALDAD DE GÉNERO EN LAS UNIVERSIDADES Y EN LA INVESTIGACIÓN ACADÉMICA}

El debate acerca de la escasa participación de las mujeres en los ámbitos académicos y científicos y las políticas para superarla tiene una larga trayectoria (Rees, 2002 , 2005). En los años 80, el diagnóstico mostraba sorpresa por la escasez de mujeres y se centraba en la explicación de los factores que influyen en la elección de estudios y carreras por parte de las chicas (profesores; padres y madres; compañeros). Se desarrollaron conceptos claves como socialización de género (Eccles et al., 1999) y tubería que gotea (leaky pipeline, Berryman, 1983) para interpretar lo que entonces muchos consideraban como un déficit de las mujeres. Las políticas se centraban en la igualdad de oportunidades (apoyo a las mujeres individualmente) y la lucha contra los estereotipos.

En los años 90 el diagnóstico avanzó asumiendo que no se puede pedir a las mujeres que se adapten a un modelo masculino de universidad y ciencia, porque el problema no está en ellas; el problema está en cómo se organizan los empleos académicos y científicos (déficit de las instituciones). El análisis se centró en la discriminación explícita e implícita en las organizaciones y profesiones académicas y científicas. La publicación clave fue "Sexism in peer-review system" de Wennerảs y Wold, 1997. Las políticas se orientaron a fomentar la atracción, entrada, promoción y retención de las mujeres en la carrera académica con medidas de acción positiva (cuotas) y desde 1995 (Conferencia de Pekín), de transversalidad de género (sistemas de incentivos a la incorporación y permanencia de las mujeres).

Ya en los 2000, el diagnóstico sobre el déficit de las instituciones académicas 
profundizó en el análisis de las normas, valores y estándares, implícitos y aparentemente neutros, que rigen su funcionamiento, como la construcción sesgada del concepto de excelencia (European Commission, 2004; Van den Brink y Benschop, 2012). Asimismo, la perspectiva del curso de la vida (life course), que aporta la consideración de la interacción entre lo institucional y lo personal a la hora de explicar el abandono de la carrera académica por parte de las mujeres que se refleja en la tubería que gotea (Castaño y Webster, 2011; Xie y Shauman, 2003). Las políticas se centraban en la necesidad del cambio estructural y cultural en las universidades y las instituciones científicas (European Commission, 2011).

Finalizada la primera década del siglo XXI, el Meta-Análisis sobre igualdad de género en la ciencia (Caprile, 2012) dedicó un capítulo específico (Castaño et al., 2010) a la revisión de la literatura y la investigación sobre políticas de igualdad de género en la ciencia y la educación superior. El balance muestra el florecimiento de las políticas de género en los países con sistemas de ciencia, innovación y educación superior más avanzados. Constata, sin embargo, que no se cuenta con evidencias científicas suficientes acerca de la efectividad de las mismas. Abunda la literatura que expone las políticas, pero es escasa la dedicada a su evaluación y está más centrada en casos individuales (programas de apoyo a grupos de mujeres específicos) que en análisis comparativos entre países o en estudios longitudinales que permitan captar los efectos a largo plazo de las políticas de igualdad. Las medidas de apoyo individual dan buen resultado para las mujeres beneficiarias, pero no cambian las instituciones ni las culturas, por lo que los pequeños avances conseguidos no se consolidan. Por ello en sus conclusiones se resalta la importancia del cambio institucional y cultural y el papel clave de los grupos representativos de las mujeres y de los organismos de igualdad.

Entre los factores del contexto universitario que afectan a la eficacia de las políticas de igualdad de género, el Meta-Analisis llama la atención sobre el hecho de que en la mayoría de los países estas políticas se han puesto en marcha en paralelo con los procesos de reformas universitarias orientados a la introducción de la lógica del mercado en la gestión de la educación superior (New Public Management) y en paralelo, asimismo, al proceso de equiparación de los distintos sistemas universitarios europeos (Declaración de Bolonia de 1999 sobre la creación del Espacio Europeo de Educación Superior, Enders et al., 2011 y 2012; Faber and Westerheijden, 2011). Ambos procesos han introducido mayor transparencia y mecanismos de competitividad y control empresarial en las universidades, poniendo en cuestión los sistemas tradicionales de producción y reproducción del poder masculino. Este cuestionamiento puede favorecer la equidad si incorpora objetivos de inclusión de mujeres e igualdad de género de forma explícita a los objetivos perseguidos con las reformas y a los criterios de evaluación de la calidad académica, teniendo en cuenta lo apuntado sobre la importancia del talento femenino y la excelencia inclusiva (Williams et al., 2005; Zippel et al., 2016)

Los análisis de las experiencias de distintos países y universidades muestran sin embargo resultados ambivalentes. Por una parte, se introducen objetivos de incrementar el número de mujeres catedráticas e investigadoras, se elaboran planes de igualdad y se crean organismos de igualdad; por otra parte, la equidad de género puede 
convertirse en un objetivo inalcanzable, entre la retórica de la igualdad y el rechazo de la perspectiva de género; entre el discurso grandilocuente que reafirma el compromiso con la igualdad, a la vez que en la práctica se identifica a la mujer con la familia y las políticas de igualdad se centran en la conciliación laboral-familiar para las mujeres, evitando poner en cuestión las estructuras de poder y las prácticas masculinas.

\section{LA REFORMA DE LA EDUCACIÓN SUPERIOR EN PERSPECTIVA DE GÉNERO}

Las reformas de la educación superior bajo la influencia del New Public Management ${ }^{1}$, introducen estrategias de gestión empresarial en las relativamente independientes y autogobernadas "torres de marfil" (Áreas de Conocimiento, Departamentos, Cátedras) que existen por doquier en las instituciones universitarias y científicas. El New Public Management (NPM) se ofrece como solución a la supuesta ineficiencia y burocratización del aparato del Estado introduciendo la lógica del mercado en el sector público de no mercado (Hood, 1991). En el caso de las universidades públicas, frente a la anterior dependencia directa de la asignación del presupuesto por parte del Estado, se trata de promover la eficiencia de la enseñanza y la investigación por la vía de la competencia por la obtención de fondos públicos mientras mejor posicionada esté cada universidad en los rankings internacionales más fondos le concederá el gobierno correspondiente-. Competencia entre universidades, competencia entre el profesorado, competencia entre el personal de investigación, como herramientas para impulsar la mejora de resultados de la docencia y la investigación. La autonomía relativa de la que gozaban las universidades, con fondos proporcionados por el Estado y sistemas de decisión descentralizados, que respetaban la libertad para crear conocimiento y transmitirlo, se enmarcan ahora en programas negociados con el gobierno, con objetivos comprometidos e incentivos acordados, con sistemas regulados de evaluación e indicadores de resultados que permitan monitorizar el progreso hacia las metas establecidas.

De esta manera, las universidades se convierten en organizaciones gestionadas a partir de los principios de mercado, competencia, elección individual, responsabilidad y eficiencia (Barry et al., 2001; Bolden et al., 2008; Deem et al., 2007; Zippel et al, 2016; Slaughter y Leslie, 1997). Todos estos cambios afectan a la relación entre la universidad y la sociedad. El conocimiento aplicado adquiere protagonismo, porque la misión de la universidad se amplía, desde el énfasis anterior en la creación y difusión de conocimiento en régimen de libertad y autonomía académica, hacia la insistencia actual en la contribución a la creación de tejido empresarial y al desarrollo de la economía y la sociedad de la información competitivas. Por su parte, la enseñanza y el

\footnotetext{
${ }^{1}$ El NPM o nueva gestión pública (NGP), pretende modernizar y hacer más eficaz el sector público orientando su gestión con criterios de mercado, introduciendo las $3 \mathrm{M}$ : Mercado, Managers y Medición. Hood, C. (1991). "A public management for all seasons?" en Public Administration, Vol. 69 (March), pag. 3-19.
} 
aprendizaje se convierten en instrumentales y vocacionales, con el objetivo directo de mejorar la empleabilidad del estudiantado, ahora clientela. Lo anterior lleva a establecer una cultura de control empresarial que prima el resultado cuantificable de la docencia y la investigación, con herramientas de transparencia y rendición de cuentas (accountability) en el marco de un sistema formalizado de aseguramiento de la calidad (quality assessment). ¿Cuántos estudiantes consigue cada docente, cada grado $\mathrm{y}$ master? ¿Qué evaluaciones tienen? ¿Cuántos fondos de investigación recauda cada departamento, cada grupo de investigación, cada personal investigador? ¿Qué tipo de empleos consiguen quienes finalizan los estudios en cada universidad? Aparece una nueva jerarquía de gestión empresarial paralela a la jerarquía académica, encargada de llevar a cabo las nuevas tareas que implica la universidad corporativa.

El NPM se empezó a implantar en los años 80 en las universidades del Reino Unido, acompañado de recortes en la financiación pública, masificación del número de estudiantes y presiones crecientes para intensificar las cargas docentes e investigadoras (Barry et al., 2006; Caprile et.al., 2011) y más tarde a Australia, Nueva Zelanda y Canadá (Fiztgerald y Wilkinson, 2011). Posteriormente se ha extendido, con diferente premura e intensidad, a otros países europeos siguiendo la tendencia general a establecer un marco de competencia para la obtención de fondos públicos y la insistencia en la transparencia y los mecanismos de rendición de cuentas en la aplicación de dichos fondos. Los procesos de reestructuración se han acompañado también de la precarización del profesorado universitario, que ha abierto la puerta a una mayor incorporación de mujeres, que se concentran en las categorías académicas más inestables (Caprile y Vallés, 2010).

\section{LAS POLÍTICAS DE IGUALDAD DE GÉNERO EN EL CONTEXTO DEL NPM EN LAS UNIVERSIDADES}

Las demandas feministas de igualdad en la universidad y la investigación, reforzadas por las políticas de igualdad impulsadas por la Comisión Europea coinciden, así, con la agenda de reformas del capitalismo académico (Slaughter y Leslie, 1997), y aparecen nuevas definiciones y mediciones de la excelencia como excelencia inclusiva, que combina la competencia por el talento y el avance hacia la diversidad de género entre el estudiantado y el personal académico (Williams et al, 2005).

El impacto de género de estos cambios se observa con perspectivas diferentes desde distintos contextos nacionales (Caprile, 2012; Caprile y Vallés, 2010; Castaño et al., 2010). La transparencia constituye un reto para las jerarquías y prácticas sesgadas por género características del modelo tradicional de las universidades $\mathrm{y}$, en este sentido, se crearían condiciones para el cambio cultural hacia la igualdad, pero sus efectos de género dependen de las regulaciones nacionales. En sentido contrario, los mecanismos de competitividad y control empresariales, basados en indicadores cuantitativos, pueden reforzar las relaciones de género en la academia, ya que las mujeres están peor situadas en publicaciones y concentradas en las categorías de profesorado más inestables y precarias. El énfasis en la excelencia inclusiva y la diversidad puede restar protagonismo a las políticas de igualdad de género. 


\subsection{LA VISIÓN DESDE EL MUNDO ANGLOSAJÓN: LA DIVISIÓN DE GÉNERO DEL TRABAJO ACADÉMICO}

Los principios del capitalismo académico llevan décadas aplicándose en las universidades anglosajonas. Tal vez por ello, la investigación es muy escéptica respecto a sus impactos positivos sobre la posición de las mujeres y la igualdad de género. Por el contrario, se considera que pueden favorecer el conformismo intelectual, exacerbar la competencia a nivel individual y ralentizar el progreso hacia la igualdad de género, ya que refuerzan las jerarquías, el sesgo masculino de la universidad y las desventajas para las mujeres. (Bagilhole, 2002; Bagilhole y Robinson, 1997; Barry et al. 2006; Blackmore y Sachs, 2007; Danowitz Sagaria 2007; Deem et al., 2007; Fiztgerald y Wilkinson, 2010; Heward y Taylor, 1993; McTavish y Miller 2009; Morley, 1999; Müller, 2006; Saunderson, 2003; Thomas y Davies, 2002; Williamson y Busy, 1997).

Tras décadas de legislación de igualdad de oportunidades, la investigación constata la diferencia entre la igualdad de trato y la igualdad de resultados. En ausencia de cambios reales, parece que las políticas de equidad hubieran servido para perpetuar involuntariamente discursos y prácticas que refuerzan la desigualdad (Blackmore y Sachs, 2007). La escasez de mujeres en puestos de máxima responsabilidad, pese al incremento del número de mujeres entre el profesorado y el estudiantado, se considera una consecuencia directa del hecho de que el NPM enmascara la desigualdad de género en las universidades, con la ayuda del discurso de la elección individual que sirve para justificar la persistencia de las brechas de género (Danowitz Sagaria, 2007).

Para el caso del Reino Unido, distintas autoras reconocen (McTavish y Miller 2009; Müller, 2006; Saunderson, 2003) que las reformas y reestructuraciones, la transparencia y rendición de cuentas, incrementan las oportunidades de acceso, para las mujeres, pero la persistencia del sesgo masculino en las prácticas cotidianas, y particularmente los nuevos criterios de excelencia y mercantilización, vuelven a sesgar por género lo nuevo. Aunque las mujeres han avanzado, las características, valores y prácticas laborales patriarcales permanecen intactas e incuestionadas.

También desde Australia, con abundante producción científica tras veinte años de políticas de igualdad en las universidades, los resultados parecen decepcionantes. Distintas autoras advierten que, con la imposición del NPM se favorece un tratamiento retórico de la problemática de la igualdad, que hace parecer que ésta ya se ha alcanzado, pero en realidad no se avanza (Fiztgerald y Wilkinson, 2010; White, 2003). Se habla de mitos (Probert, 2005) y espejismos (Fiztgerald y Wilkinson, 2010) porque la presencia creciente de mujeres en la academia se utiliza como argumento para pretender que la batalla de la igualdad está ganada: las mujeres tienen igualdad de oportunidades de acceso y promoción y los discursos de la elección individual explican la persistencia de la brecha de género. Se advierte erosión y desgaste de las políticas de igualdad de género como consecuencia de las reformas. Por ejemplo, las unidades de igualdad son incorporadas a unidades de diversidad y la igualdad de género se convierte en responsabilidad de todos y de ninguno, se queda en la periferia de la 
organización (Fiztgerald y Wilkinson, 2010) y se asigna como responsabilidad exclusiva a las mujeres. Esto también se aprecia en todo el entorno de los países anglosajones (Danowitz Sagaria, 2007; Heijistra et al., 2016).

Una encuesta de Bagilhole y Robinson (1997), mostraba la falta de efectividad de las medidas de igualdad de género: aunque el $90 \%$ de las instituciones de educación superior del Reino Unido contaban con políticas de igualdad, el 80\% no habían tomado ninguna medida tras hacer el diagnóstico, y las acciones positivas se limitaban a formación específica para mujeres y publicidad. En otro estudio de Bagilhole (2002) basado en entrevistas a profesorado de todos los niveles universitarios, se concluye que la cultura competitiva de individualismo aislacionista y los estilos de gestión de universidades, facultades y departamentos, hace virtualmente imposible alcanzar la igualdad de oportunidades. La adopción formal de las políticas de igualdad sin una estrategia clara de aplicación sería contraproducente según Bagilhole, porque lleva a la convicción de que la igualdad de género ya existe. Los efectos de las políticas son marginales y la retórica de la igualdad inefectiva, porque persiste la confianza en que la determinación del mérito académico individual es objetiva y neutral como base de acceso a una jerarquía basada en el prestigio, que sin embargo favorece a los hombres (Heward y Taylor, 1993; Williamson y Busy, 1997).

Una problemática de gran interés en el ámbito anglosajón es la referida al impacto del NPM sobre el aumento de la intensidad del trabajo, el estrés de la vida académica y el desequilibrio trabajo-vida privada. Thomas y Davies (2002), demuestran que la intensificación del trabajo bajo las reformas del NPM introduce una enorme presión sobre el personal académico, que trabaja horas y horas en condiciones de dedicación y celo competitivo extremos. El trabajo académico se convierte en una actividad productiva altamente competitiva, orientada a generar productos claramente identificables desde el punto de vista cuantitativo. Esto refuerza un estilo de carrera basada en el modelo masculino, que no tiene en cuenta los compromisos domésticos ni otras opciones, como por ejemplo carreras más lentas o carreras que combinen la gestión y la docencia con la investigación.

Probert (2004) demuestra para Australia que la intensificación del trabajo académico es mayor entre las mujeres (que dedican mucho tiempo a la enseñanza en primer curso, la mentoría y el bienestar de los estudiantes y de toda la comunidad universitaria) que entre los hombres (que supervisan a los graduados, organizan conferencias y hacen consultoría e investigación). Advierte que, al ser la docencia más difícil de posponer que la investigación, esto da lugar a situaciones de mujeres sobresaturadas, que trabajan fuera del horario en tareas de responsabilidad administrativa y de atención a estudiantes, que no avanzan en su carrera académica por falta de tiempo para dedicar a la investigación y publicación. Es lo que se denomina el techo del cuidado (care ceiling, Grummell et al., 2009).

Un análisis comparativo del impacto del NPM en Suecia y Reino unido (Barry et al, 2006) muestra que, a pesar de las diferencias culturales, en ambos países las mujeres experimentan muchas más dificultades que sus colegas varones para hacer frente a sus compromisos de trabajo en la universidad, lo que implica consecuencias graves de mala salud, cambio de puestos de trabajo y abandono de la investigación. 
Distintas autoras (Blackmore y Sachs, 2007; Deem et al., 2007) analizan cómo las reformas y reestructuraciones inspiradas en el NPM abren nuevas oportunidades de ascenso y carrera para las mujeres en las universidades y muestran sus experiencias como gestoras y agentes de cambio, mentoras y líderes. Las universidades proclaman su compromiso con la igualdad mediante la promoción de mujeres a los niveles más altos de gestión, pero hay una re-tradicionalización de las relaciones de género: gestión feminizada e investigación masculinizada. Fiztgerald y Wilkinson (2010) muestran para el caso de Nueva Zelanda, con datos de 2000-2007, que hay más mujeres en puestos de responsabilidad porque los hombres se van a la investigación y dejan la gestión para las mujeres.

Se reproduce la división sexual del trabajo en la universidad, con el dominio numérico de las mujeres en los escalones más bajos y en áreas y disciplinas poco prestigiadas (Glazer-Raymo, 2007), y con trayectorias de carrera diferentes para unos y otras. Los hombres mantienen para sí las tareas más prestigiosas de investigación, gobernanza, estrategia, consultoría y relaciones con empresas (Fiztgerald y Wilkinson, 2010; Probert, 2005; Wilson et al., 2010) y las mujeres se hacen cargo de la enseñanza en los primeros cursos y la atención a los estudiantes, que tienen un elevado componente de trabajo emocional (cuidado del rebaño o pastoral care, en términos de Probert, 2005).

Se hacen cargo también de los nuevos puestos que el NPM ha creado, dedicados a desarrollo organizativo y cambio institucional, como las auditorías de calidad. Son puestos que implican un enorme volumen de trabajo administrativo y de atención al público, que requieren habilidades de comunicación y emocionales, pero desvalorizados y considerados como trabajo de ama de llaves institucional (institutional housekeeping, Dearlove, 2002; Deem et al., 2007; Morley 2005; Simpson y Fiztgerald, 2014). Algo similar ocurre con los asuntos sociales y la igualdad de género, tareas consideradas de exclusiva responsabilidad de las mujeres y trabajo de ama de llaves académica, (academic housework, Bird et al., 2004; Heijistra et al., 2016).

Por último, los procesos de reestructuración de las universidades implican reducciones de tamaño y cierres de escuelas y facultades, fusiones de departamentos, que con frecuencia conllevan despidos de profesores y trabajadores administrativos. Estos puestos de problemática gestión, que curiosamente también son llevados a cabo por mujeres, son denominados por Ryan y Haslam (2005) como acantilados de cristal (glass cliffs) por el estrés que implican, además de incorporar un considerable volumen de trabajo emocional (Heijistra et al., 2016).

Las universidades se apoyan en el trabajo emocional invisible de las mujeres, también en los puestos de gestión de máximo nivel. Se ejerce una enorme presión para que asuman el liderazgo en los puestos menos deseables y también como decanas y jefas de departamento. Probert (2004) demostró que las mujeres dedican mucho más tiempo al trabajo de cuidado pastoral y gestión académica e institucional, mientras que los hombres se dedican a consultoría e investigación, posgrado y organización de conferencias, esto tiene efectos acumulativos positivos para la carrera académica de los hombres y negativos para la de las mujeres. Ellas están atrapadas entre las presiones 
corporativas, las demandas de la universidad y sus propias responsabilidades como profesoras e investigadoras, participación en comisiones de igualdad y calidad, mientras que la productividad investigadora no es negociable a la hora de la carrera académica (Probert, 2005). Mujeres con más carga de trabajo que los hombres, mujeres quemadas (burnout en términos de Blackmore y Sachs, 2007) pero de las que se espera que se dediquen al trabajo de gestión emocional de la organización, como una extensión natural de sus tareas tradicionales como esposas y madres. Son las trabajadoras invisibles que hacen funcionar la universidad (Stanley, 1997).

\subsection{LA VISIÓN DESDE ALEMANIA Y AUSTRIA: FINANCIACIÓN LIGADA A LA IGUALDAD DE GÉNERO}

En Alemania y Austria, países líderes en innovación (European Commission, Benchmarking Report, 2008) la reforma de la educación superior ha ligado la financiación de las universidades al logro de objetivos de productividad y calidad, pero también de incorporación de mujeres al profesorado y de medidas de igualdad de género. La abundante literatura en idioma alemán recogida en el Meta Análisis (Castaño et al., 2010) que sería prolijo citar aquí, discute las ventajas de las políticas de promoción de las mujeres a través del presupuesto, que fomentan el interés de los responsables universitarios por avanzar en la igualdad. La investigación académica se pregunta si estas medidas de transparencia y rendición de cuentas, pueden facilitar el establecimiento de vínculos sistemáticos entre la reforma universitaria y las políticas de igualdad de género. Rothe et al. (2008), comparan la introducción de los presupuestos de género en tres universidades, de Alemania, Austria y Polonia y encuentran que la transparencia, el gobierno por objetivos y el control financiero constituyen puntos de partida interesantes, pero la concentración de poder en el nivel de gestión más alto implica que en la medida en que las consideraciones financieras que se presentan como decisiones "puramente técnicas" y sin consecuencias políticastienen preferencia sobre todo lo demás, las políticas de igualdad son mucho más fácilmente prescindibles que anteriormente, Lo anterior, unido a la ausencia de conciencia de género de los gestores financieros, hace que se pierda el potencial de cambio de los presupuestos.

En Alemania se pusieron en marcha en 2005 varias iniciativas orientadas a la incorporación del talento de las mujeres, motivadas por cierta presión política -solo una de las 100 universidades alemanas había entrado en el Ranking de Shangai 2003 de las Top 50 Universidades (Zippell et al., 2016). La Universities Excellence Initiative estableció que los contratos entre el Estado y las universidades para la financiación pública de las mismas, los compromisos de resultados y calidad han de incorporar objetivos de igualdad de género, diversidad, mujeres y minorías. Los indicadores de rendimiento incluyen compromisos explícitos sobre indicadores de género, como por ejemplo la proporción de plazas de profesorado y cátedras de sexo femenino (Roloff, 2007; Castaño et al., 2010; Müller et al., 2011; Zippel et al., 2016). La igualdad de género ya no sería una cuestión de retórica inclusiva, sino un criterio de financiación de máximo interés para los gestores. 
En 2008 se establecieron nuevos criterios de selección en las convocatorias competitivas, que incluían requisitos para que la investigación académica tuviera en cuenta el género, tanto incorporando mujeres a los equipos, como por la consideración de los factores relacionados con sexo y género en el contenido, objetivos y resultados de las investigaciones. Sin embargo, como la investigación de Lipinsky y Samjeske (2012) muestra, el género aparecía como un criterio politico externo impuesto a los evaluadores, al que los investigadores principales respondían de manera burocrática utilizando plantillas estandarizadas, pero sin considerarlo en el contenido de la investigación.

Los informes del Consejo Alemán de Ciencias y Humanidades (Wissenchafstrat, 2007), consideraban los avances insuficientes y proponían nuevas medidas de apoyo individual a las mujeres. Sin embargo, la mayoría de las acciones puestas en marcha se orientaron a mitigar conflictos familia-trabajo, más que a fomentar el desarrollo organizativo de la igualdad de género (Zippel et al., 2016).

En el caso de Alemania, a pesar de las limitaciones del discurso neoliberal -la incorporación de mujeres como un recurso competitivo - estas medidas han servido para que la igualdad de género avance y han reforzado el papel institucional de los organismos de igualdad de las universidades participantes. Persisten, sin embargo, dos problemas de efectividad. Por una parte, todo el entramado se basa en herramientas de autorregulación -la participación es voluntaria- que ofrecen a las universidades la oportunidad de demostrar su compromiso con la igualdad de género -número de medidas; número de profesoras contratadas- como una herramienta más para mejorar en los rankings. Por otra parte, la mayoría de las medidas adoptadas afirman que su objetivo es hacer sus instituciones más amigables con la familia (family friendly) y solo unas pocas se refieren a la institucionalización del género en la docencia y la investigación ${ }^{2}$.

La preferencia por el apoyo a las tareas de cuidados que realizan las madres como herramienta principal para resolver las desigualdades de género en Alemania, es analizada por Zippel et al. (2016), como un proceso de vernacularización de las políticas de género de la UE, que se lleva a cabo en el seno de lo que estas autoras denominan triángulo de terciopelo: expertas/os académicas feministas, organismos de igualdad de las universidades y agencias del gobierno que financian las universidades y la investigación. Lo positivo de este marco de actuación es que se ha construido un consenso normativo que entiende la igualdad de género como una forma de excelencia (excelencia inclusiva) que favorece la competitividad, y por tanto, la ausencia de mujeres sería incompatible con la excelencia. En todo caso, persisten resistencias a considerar el sexo y el género en el diseño y evaluación de la investigación como señalan Lipinsky y Samjeske (2012). Lo negativo es que se despolitiza la igualdad de género, alejándola del marco de la justicia social e incluyéndola en el marco de la gestión empresarial. Las universidades alemanas, además, han interpretado que para alcanzar la igualdad de género lo necesario era convertir sus instituciones en más

\footnotetext{
${ }^{2}$ Las universidades pueden pasar cada dos años una auditoria family frinedliness que también pasan las empresas y centros de investigación. (Zippel et al., 2015).
} 
familiares (family friendly). La agenda de la igualdad se va estrechando hasta convertirse en un plan de conciliación trabajo-familia, con los Rectorados mostrando orgullosos las guarderías que han construido. El acento en lo familiar resta importancia a otros problemas más difíciles de superar, como la evaluación sesgada o las redes que excluyen a las mujeres y reproducen la desigualdad. Asímismo, en la investigación, los recursos se dedican de forma desproporcionada a incluir mujeres en los campos de la ciencia y la tecnología, mientras que se financian pocos programas de investigación o escuelas de formación sobre feminismo y género (Zippel et al., 2016).

El sistema austriaco de igualdad de género en las universidades se basa en tres pilares (Wroblenski y Leitner, 2011): la implicación activa de las mujeres académicas e investigadoras en el diseño de las medidas de igualdad; el diseño de las políticas a partir de "evidencias empíricas" - diagnósticos basados en resultados de investigación- y la introducción de los "estudios de género" como área de investigación; $\mathrm{y}$, finalmente, un objetivo doble y cuantificado de promover a las mujeres individualmente y promover también la igualdad de género. Con la Ley de Universidades de 2002, cada universidad ha de establecer un plan de igualdad y tener evaluaciones de género positivas para mantener y aumentar sus recursos financieros. El sistema asume que el aumento de la participación de las mujeres en la gestión de las universidades pondrá en cuestión y cambiará las normas de dominación masculina provocando el cambio cultural, lo que ha servido para justificar la introducción en 2009 de una cuota de mujeres del $40 \%$ para los órganos de toma de decisión de las universidades.

Wroblenski (2014) argumenta que la cuota del 40\% solo se ha respetado en los órganos que tienen gran visibilidad pública (Rectorado y Vicerectorados), y donde son obligatoria por ley -comisiones de contratación y promoción. Sin embargo, allí donde la cuota depende de la conciencia de género, ha habido pocos cambios.

La investigación sobre el caso Austríaco muestra dudas sobre si los nuevos estilos gerenciales, a pesar de haber contribuido a reducir la influencia de las redes informales de poder, implican en realidad un fortalecimiento de las posiciones de alto nivel, lo que reduciría las posibilidades de participación democrática de los rangos inferiores de la jerarquía universitaria, donde se encuentran proporcionalmente más mujeres (Pellert and Gindl, 2007; Rothe et al., 2008; Wroblenski y Leitner, 2011). La presencia creciente de mujeres entre el profesorado de las universidades y mayoritaria entre el alumnado, no ha implicado la puesta en cuestión del ideal normativo de científico, porque tanto la lógica del mercado como la lógica académica asumen que la realidad es desigual y dan prioridad la eficiencia. De esta manera, la equidad se queda sin espacio y se evapora el objetivo de superar los sesgos, la desigualdad y la discriminación de género.

\subsection{LA GESTIÓN POLÍTICA DE LA DESIGUALDAD: EQUIDAD O DIVERSIDAD}

Con la aproximación neoliberal propia del NPM las desventajas y desigualdades que sufren las mujeres se consideran un problema político que ha de ser gestionado. 
Zippel et al. (2016), utilizan el concepto de capitalismo académico de Slaughter y Larry (1999, gobierno de los mánagers y comercialización del conocimiento), para contraponer los dos modelos de gestionar el género en un entorno caracterizado por la rendición de cuentas (accountability): la aproximación Anglo-Americana de gestión de la diversidad (diversity management, inspirada en el modelo corporativo empresarial privado) frente a la aproximación predominante en la Unión Europea de transversalidad (gender mainstreaming, con gestión institucionalizada del género por parte del Estado, Danowitz Sagaria, 2007). Los dos modelos presentan discursos específicos respecto al mérito. La rendición de cuentas (accountability) sintetiza el discurso neoliberal del gobierno de los gestores científicos (basado en la productividad, la eficiencia y el control), mientras que el discurso académico tradicional de la excelencia científica todavía se sustenta en valores de individualismo, modernidad, democracia y humanismo. Sin embargo, en la actualidad, ambos discursos se superponen y configuran de forma conjunta las políticas de género de las universidades, orientadas a la creación y uso eficiente de recursos humanos para hacer más competitivas las economías del conocimiento nacionales. No se ponen en cuestión las medidas de productividad académica y las mediciones del mérito vigentes, que acumulan ventajas para los que ya están bien situados, los hombres, de manera que el capitalismo académico refuerza, en lugar de retar, los sesgos de género. Transversalidad y gestión de la diversidad aparecen, así como estrategias de gestión de la igualdad que se apoyan en métricas de éxito cuantitativas y utilizan la competitividad por ascender en los rankings como elemento motivador del esfuerzo, pero nunca critican las jerarquías previamente existentes. Como la competencia es el principio motivador universal, las ciencias útiles -ciencias puras, tecnología, ingeniería, matemáticas- son prioritarias, mientras que las ciencias sociales quedan relegadas y la investigación feminista y de género no siempre es considerada científica.

Aunque la diversidad es positiva y necesaria, y es importante diseñar y aplicar políticas que la estimulen y la respeten, la academia depende todavía de normas y valores masculinos, y en las universidades el conflicto de poder central se establece en términos de patriarcado, relación de dominación de los hombres respecto a las mujeres en la que éstas son consideradas todavía como "los otros" (Acker, 1995). Bajo el NPM, se trata de sustituir la equidad de género por las políticas de diversidad, aplicando nociones esencialistas -en términos de diferencias individuales, no de relaciones de dominación estructurales- como ventajas para el prestigio de la organización (Blackmore, 2002). El problema para las mujeres y para la equidad de género es que el objetivo de la diversidad es mejorar a algunos de los que están en desventaja, en lugar de profundizar en cómo funcionan los privilegios y tratar de desactivarlos. Se acepta que la realidad es desigual y no se analiza por qué hay barreras y privilegios, se trata simplemente de suavizarlos. (Balgihole, 2002; Blackmore y Sachs, 2007; Fiztgerald, 2009; Morley, 1999). La erosión de las políticas de igualdad de género avanza conforme se asume que las mujeres pueden adaptarse a los valores de individualismo y mercado, porque pueden elegir-. El mito de la libre elección sirve para justificar las desigualdades como diferencias (Deem et al., 2007). El género desaparece poco a poco del escenario y las unidades de igualdad son incorporadas a unidades de diversidad. La igualdad de género se convierte en responsabilidad de todos y de ninguno, en la 
periferia de las organizaciones (Blackmore, 1997) y asignada a las mujeres de forma exclusiva, como parte del institutional housekeeping (Bird et al., 2004; Heijistra et al., 2016). De la igualdad solo queda el espejismo numérico, el aumento del número de mujeres (Fiztgerald y Wilkinson, 2010).

\section{CONCLUSIONES}

La literatura y la investigación reseñadas en las páginas anteriores muestran que las políticas de igualdad de género que se han aplicado en las universidades bajo el New Public Management tienen resultados ambivalentes. Por una parte, se va incrementando, aunque lentamente, el número de mujeres catedráticas e investigadoras, se elaboran planes de igualdad, se crean organismos de igualdad y se abren nuevas oportunidades de ascenso y carrera para las mujeres. Por otra parte, se reproduce la división de género del trabajo, con las mujeres en los nuevos puestos de atención a los estudiantes y al cuidado del desarrollo organizativo e institucional (auditorías de calidad; bienestar social; igualdad). Finalmente, la equidad de género parece un objetivo inalcanzable, entre la retórica grandilocuente que afirma el compromiso con la igualdad, y las prácticas que identifican a las mujeres con la familia.

La literatura que analiza la experiencia del Reino Unido y Australia alerta sobre cambios más retóricos que reales, y prácticas que ocultan la desigualdad. La adopción formal de políticas de igualdad sin una estrategia clara de aplicación sería contraproducente, porque lleva a la convicción de que la igualdad de oportunidades ya existe.

En Alemania y Austria, la reforma de la educación superior ha ligado la financiación de las universidades al logro de objetivos de incorporación de mujeres al profesorado La efectividad de las reformas alemanas se cuestiona porque su aplicación es voluntaria $\mathrm{y}$, por otra parte, la mayoría de las acciones de igualdad puestas en marcha se orientan facilitar la conciliación familia-trabajo, evitando abordar problemas de fondo como la evaluación sesgada o las redes que excluyen a las mujeres y reproducen la desigualdad.

El sistema universitario austriaco apuesta por el aumento de la participación de las mujeres en la gestión de las universidades para cuestionar las normas de dominación masculina y provocar el cambio cultural, pero ello no ha implicado, por el momento, la puesta en cuestión del ideal normativo de científico, porque tanto la lógica del mercado como la lógica académica asumen que la realidad es desigual y dan prioridad la eficiencia.

Con el NPM, transversalidad y diversidad configuran de forma conjunta las políticas de igualdad de las universidades, pero se apoyan en métricas de éxito cuantitativas, mientras que nunca critican las jerarquías previamente existentes. Se corre el riesgo de sustituir las políticas igualdad de género por políticas de diversidad. De la igualdad solo quedaría el espejismo numérico -el aumento del número de mujeres- y el mito de la libre elección para justificar las desigualdades como diferencias. 


\section{REFERENCIAS BIBLIOGRÁFICAS}

ACKER, SANDRA (1995): Carry on Caring: the work of women teachers. British Journal of Sociology of Education, 16(1), 21-36.

BAGILHOLE, BARBARA (2002): Academia and the Reproduction of Unequal Opportunities for Women, Science Studies, 15 (1), 46-60.

BAGILHOLE, BARBARA \& ROBINSON, E (1997): A report on policies and practices on equal opportunities in employment in universities and colleges in higher education, Commission on University Career Opportunity, Committee of Vice-Chancellors and Principals of the Universities of the United Kingdom, London.

BARRY, JIM; BERG, ELISABETH AND CHANDLER, JOHN (2006): Academic Shape Shifting: Gender, Management and Identities in Sweden and England, Organization, 13:2, 275-298.

BERRYMAN, S. (1983): Who will Do Science? Minority and Female Attainment of Science and Mathematics Degrees: Trends and Causes, Rockefeller Foundation, New York.

BIRD, SHARON; LITT, JACQUELYN, S. \& WANG, YONG (2004): Creating Status of Women Reports: Institutional Housekeeping as'Women's Work. NWSA Journal, 16: 194-206.

BLACKMORE, JILL (2002): Globalisation and the Restructuring of Higher Education for New Knowledge Economies: New Dangers or Old Habits Troubling Gender Equity Work in Universities, Higher Education Quarterly, 56 (4) 181-199.

BLACKMORE, JILL \& SACHS, JUDYTH (2007): Performing and Reforming Leaders: Gender, Educational Restructuring, and Organizational Change. Albany: State University of New York Press.

BOLDEN, RICHARD; PETROV, GEORGY \& GOSLING, JONATHAN. (2008): Developing collective leadership in higher education, final Report. London: Leadership Foundation for Higher Education.

8th Conference on Gender Equality in Higher Education, Book of Abstracts, Vienna University of Technology. September 3-5, 2014. Available https://gender2014.conf.tuwien.ac.at/fileadmin/t/gender2014/Gender_Conference Abstracts_A4_280814.pdf 
CAPRILE , M.ARIA (Coord.), ADDIS, ELISABETTA; CASTAÑO, CECILIA; MEULDERS, DANIELE; MÜLLER, JÖRG; PLASMAN, RORBERT, SCHIEBINGER, LONDA \& VALLÈS, NURIA (Eds.) (2012): Meta-analysis gender and science research: Synthesis report. Luxembourg: Publications Office of the European Union.

CAPRILE, MARIA \& VALLÈS, NÚRIA (2010): Science as a labour activity. Metaanalysis of gender and science research - Topic report. Available: http://www.genderandscience.org/doc/TR4_Labour.pdf

CAPRILE, MARIA; MEULDERS, DANIÈLE, MEULDERS; O’ DORCHAI, SÍLE \& VALLÉS, NÚRIA (2011): Introduction to Special Issue. Gender and Science: Old Challenges, New Approaches, Brussels Economic Review, Vol.54 (2/3).

CASTAÑO, CECILIA,; MÜLLER, JÖRG; PALMÉN, RACHEL; GONZÁLEZ RAMOS, ANA (2010): Policy towards gender equity in science and research. Meta-analysis of gender and science research. Topic report. http://www.genderandscience.org/doc/TR7_Policies.pdf

CASTAÑO, CECILIA \& WEBSTER, JULIET (2011): Understanding women's presence in ICT: the life course perspective. International Journal of Gender, Science and Technology, 3(2), 364-386.

COMISIÓN EUROPEA (2000): Estrategia de Lisboa para el crecimiento y el empleo. Available: ec.europa.eu/europe2000/index es.htm

COMISIÓN EUROPEA (2010): Europa 2020: La estrategia europea para el crecimiento. Available: ec.europa.eu/europe2020/index_es.htm

DANOWITZ SAGARIA, MARY ANN (2007): “Gender Equality as Organizational Change Frames, Challenges, and Strategies in the European Union and the United States", en Grenz, S., Kortendiek, B., Kriszio, M. \& Löther, A. (eds.) Gender Equality Programes in Higher Education. International Perspectives. 87-100 Wiesbaden, Verlag für Sozialwissenchaften

DANOWITZ SAGARIA, MARY ANN (Ed.). (2007): Women, Universities and Change: Gender Equality in the European Union and the U.S. London, Palgrave Macmillan

DAVIS, G (2005): Optimizing the Postdoctoral Experience: An Empirical Approach (Working Paper). Research Triangle Park, NC: Sigma Xi, The Scientific Research Society. 
DEARLOVE, JOHN (2002): A Continuing Role For Academics: The Governance of UK Universities in the Post-Dearing Era, Higher Education Quarterly, 56 (3):257275.

DEEM, ROSEMARY, HILLYARD, SAM \& REED, MICHAEL (2007): Knowledge, Higher Education, and the New Managerialism. The Changing Management of UK Universities. Oxford: University Press.

ECCLES, JAQUELYNNE S.; BARBER, BONNIE \& JOZEFOWICZ, DEBRA (1999): Linking gender to educational, occupational, and recreational choices: Applying the Eccles et al. model of achievement-related choices. En W. B. Swann, J. H. Langlois, \& L. A. Gilbert (Eds.), Sexism and stereotypes in modern society: The gender science of Janet Taylor Spence (pp. 153-192). Washington DC: American Psychological Association.

ENDERS, JÜNGEN; DE BOER, HARRY DE \& WESTERHEIJDEN, DON (eds.). (2011): Reform of Higher Education in Europe. Boston: Sense Publisher.

ENDERS, JÜNGEN, DE BOER, HARRY \& AND WEYER, ELKE (2012): Regulatory autonomy and performance: the reform of higher education re-visited, Higher Education, Doi 10.1007/s10734-012-9578-4, published on line 16 october 2012.

ETAN EXPERT WORKING GROUP (2000): Science Policies in the European Union. Promoting excellence trough mainstreaming gender equality Available: ftp://ftp.cordis.europa.eu/pub/improving/docs/g_wo etan_en_200101.pdf as consult July 21st, 2016.

EUROPEAN COMMISSION (2004): Gender and Excellence in the Making Directorate for Research.

EUROPEAN COMMISSION (2008): Information Society Benchmarking Report. Brussels: European Commission.

EUROPEAN COMMISSION, (2011): Structural Change Report Versión española de la obra original en lengua inglesa Structural change in research institutions: Enhancing excellence, gender equality and efficiency in research and innovation, publicada por European Union. Madrid: Ministry of Science and Innovation.

Available: http://www.idi.mineco.gob.es/stfls/MICINN/Ministerio/FICHEROS/UMYC/Cam bio estructural instituciones cientificas.pdf 
EUROPEAN COMMISSION (2015): She Figures 2015. Brussels, Directorate-General for Research and Innovation.

FABER, MARIKE AND WESTERHEIJDEN, DON (2011): European Degree Structure and National Reform: Constitutive Dynamics of the Bologna Process, in Jürgen Enders, Harry de Boer, and Don Westerheijden (Eds.) (2011) Reform of Higher Education in Europe (pp. 11-28). Boston: Sense Publisher.

FÄRBER, CHRISTINE (2000): 'Das Anreizsystem Frauenförderung der Freien Universität Berlin' in Löther, A. \& Plöger, L.Kleine Verlag, Bielefeld (Eds.) Mittelvergabe \& Gleichstellungspolitik an Hochschulen (pp. 59-75).

FITZGERALD, TANYA (2009): ¿Outsiders or equals? A history of women professors at the University of New Zealand 1911-1961. Oxford: Peter Lang.

FITZGERALD, TANYA \& WILKINSON, JANE (2010): Travelling towards a mirage? Gender, leadership and higher education. Brisbane: Post Pressed.

GLAZER-RAYMO, JUDITH (2007): Gender Equality in the American research University: Renewing the Agenda for Women's Rights, en Danowitz, M. A., (ed.), Women, Universities and Change. Gender Equality in the European Union and the United States (pp. 161-178). Palgrave MacMillan, New York.

GRUMMELL, BERNIE; DEVINE, DYMPNA \& LYNCH, KATHELEEN (2009): The care-less manager: gender, care and new managerialism in higher education. Gender and Education, 21(2), 191-208

HEIJISTRA, THAMAR MELANIE; STEINTHORSDÓTTIR, FINNBORG SALOME \& EINARSDÓTTIR, THORGERDUR (2016): Academic career making and the double-edged role of academic housework, Gender and Education, published online, 62016.2 april 20 Available: http://www.tandfonline.com/doi/abs/10.1080/09540253.2016.1171825

HEWARD, C \& TAYLOR, P (1993): 'Effective and ineffective equal opportunities policies in higher education', Critical Social Policy, vol. 13, no. 37, pp.75-94.

HOOD, C. (1991): A public management for all seasons? En Public Administration, Vol 69, March. 1991, pp. 3-19.

LIPINSKY, A. AND SAMJESKE, K. (2012): Consideration of Gender Aspects in EUfunded Research Projects in Germany CEWS. 
MCTAVISH, D. \& MILLER, K. (2009): Gender Balance in Leadership?: Reform and Modernization in the UK Further Education Sector. Educational Management Administration Leadership, 37(3),350-365.

MORLEY, L. (1999): Organising feminisms. The micropolitics of the academy. Basingstoke: Macmillan Press.

MORLEY, L. (2005): 'Opportunity or exploitation? Women and quality assurance in higher education ', Gender and Education, vol. 17, no. 4, pp. 411-429.

MÜLLER, JÖRG; CASTAÑO, CECILIA; GONZÁLEZ, AANA \& PALMÉN, RACHEL (2011): Policies towards gender equality in science and research, in Brussells Economic Review 54(2/3): 295-316, Summer-Autum 2011.

MÜLLER, URSULA (2006): 'Between Change and Resistance: Gender Structures and Gender Cultures in German Institutions of Higher Education', IFF Info, Zeitschrift des Interdisziplinären Zentrums für Frauen- und Geschlechterforschung der Universität Bielefeld, vol. 23, no. 31, pp. 7-20.

PELLERT, ADA AND GINDEL, M (2007): Gender Equity and Higher Education Reform in Austria, en Danowitz Sagaria, M.A. (Ed.). Women, Universities and Change: Gender Equality in the European Union and the U.S. (pp. 61-72). London, Palgrave Macmillan

PROBERT, B. (2004): If Only It Were A Glass Ceiling: Gendered Academic Careers, en Charlesworth, S. and Fasteanu, M (Eds.). Women and Work: Current RMIT University Research. RT University, Melbourne

PROBERT, BELIDA (2005): 'I just couldn't fit it in': Gender and unequal outcomes in academic careers. Gender, Work and Organisation 12(1): 50-72.

REES, TERESA (2005): Reflections of the Uneven Development of Gender Mainstreaming in Europe, International Feminist Journal of Politics, 7 (4): 555574.

REES, TERESA (2002): National Policies on Women and Science in Europe. Directorate General for Research, Women and Science, Luxembourg: Office for official Publications of European Communities.

ROLOFF, CHRISTINE (2007): Gender Equality Challenges and Higher Education Reform: A Case Study University of Dortmund, in Sagaria, M. (ed.), Women, 
Universities, and Change. Gender Equality in the European Union and the United States. Palgrave Macmillan: New York.

ROTHE, ANDREA; ERBE, BIRGIT; FRÖHLICH, WERNER; KLATZER, ELISABETH; LAPNIEWSKA, ZAFIA; MAYRHOFER, MONIKA \& NEUMAYR, MICHAELA. (2008): Gender Budgeting as a Management Strategy for Gender Equality at Universities. Concluding Project Report, Downloaded on 23/09/2009, Available: http://www.frauenakademie.de/projekt/eu_genderbudgeting/img/FAM-GB management_conclusion_2008.pdf.

RYAN, MICHELLEAND HASLAM, ALEXANDER (2005): The glass cliff: evidence that women are over-represented in precarious leadership positions, British Journal of Management, 16 (2), 81-90.

SAUNDERSON, WENDY (2003): Women, Academia and Identity: Constructions of Equal Opportunities in the 'New Managerialism'- A Case of 'Lipstick on the Gorilla'?, Higher Education Quarterly, 56, (4), 376-406.

SIMPSON, ANDREA \& FITZGERALD, TANIA (2014): Professional knowledge workers: tensions and binaries, in Tania Fitzgerald (ed) Advancing knowledge in higher education: Universities in turbulent times. (pp. 48-65). Hershey, PA: IGI Global.

SLAUGHTER, SHEILA \& LESLIE, LARRY, LESLIE (1997): Academic capitalism: Politics, Policies and the Entrepreneurial University. Johns Hopkins UP.

STANLEY, LIZ (1997): Knowing Feminisms: On Academic Borders, Territories and Tribes. SAGE Publications: London.

THOMAS, ROBYN \& DAVIES, ANNETTE (2002): Gender and New Public Management: Reconstituting Academic Subjectivities, Gender, Work and Organization, vol. 9, no. 4, pp. 372-397.

VALIAN, VIRGINIA (1998): Why So Slow? The Advancement of Women. Cambridge, Mass.: MIT Press.

VAN DEN BRINK, MARIEKE VAN DEN \& BENSCHOP, YVONNE (2012) Slaying the Seven-Headed Dragon: The Quest for Gender Change in Academia, Gender, Work \& Organization, 19 (1), 71-92.

WENNERÅS, CHRISTINE \& WOLD, AGNES (1997): Nepotism and sexism in peerreview, Nature, 387, 341-343. 
WHITE, KATE (2003): Women and leadership in higher education in Australia. Tertiary Education and Management, 9: 45-60.

WILLIAMS, DAMON A.; BERGER, JOSEPH B. \& MCCLENDON, SHEDERICK, A. (2005): Toward a model of inclusive excellence and change in postsecondary institutions. American Association of Colleges and Universities. Available at: https://www.aacu.org/sites/default/files/files/mei/williams_et_al.pdf

WILLIAMSON, VALERIE \& BUSBY, HELEN (1997). 'Where are we now? Auditing the implementation of an equal opportunities policy 'on the ground' in a new university', Journal of Further and Higher Education, 21 (2), 251-260.

WILSON, JACQUELINE Z., MARKS, GENEE; NOONE, LYNNE AND HAMILTON-MACKENZIE, JENNIFER (2010): Retaining a foothold on the slippery paths of academia: university women, indirect discrimination, and the academic marketplace, Gender and Education 22(5):535-545.

WISSENSCHAFTSRAT (2007). Empfehlungen zur Chancengleichheit von Wissenschaftlerinnen und Wissenschaftlern. Mainz: Wissenschaftsrat. http://www.wissenschaftsrat.de/Veroffentlichungen/veroffentlich.htm

WROBLENSKI, ANGELA. (2014): Female participation in management and cultural change: precondition or high expectations?, Paper presented at the 8th International, Interdisciplinary Conference on Gender, Work and Organization (GWO) 24th-26th June 2014, Keele (UK)

WROBLENSKI, ANGELA \& LEITNER, ANDREA (2011): Equal Opportunities Policies at Austrian Universities and their Evaluación: Development, Results and Limitations. Brussells Economic Review 54(2/3), 317-340.

XIE, YU \& SHAUMAN, KIMBERLEE A. (2003): Women in Science: Career Processes and Outcomes. Harvard University Press, Cambridge: Massachusetts.

ZIPPEL, KAHRIN; FERREE, MYRA MARX \& ZIMMERMANN, KARIN (2016): Gender equality in German universities: vernacularising the battle for the best brains. Gender and Education, pp. 1-19, Published online: 29 Jan 2016: http://www.tandfonline.com/doi/abs/10.1080/09540253.2015.1123229. 